\title{
基于平行因子分析法提高近红外无创血糖校正模型稳健性的研究
}

\author{
张婉洁刘蓉* 徐可欣 \\ (天津大学 精密测试技术及仪器国家重点实验室 天津 300072)
}

\begin{abstract}
摘要 采用近红外光谱进行无创血糖检测时, 样品背景变动造成的预测集样本与校正集样本量测体系不一致的问题是 导致预测精度低的原因之一. 提出一种将母体背景作为变量引入回归建模中, 结合各个母体背景下的样本光谱信息构 建三维光谱矩阵以提高校正模型稳健性的分析方法. 将平行因子分析(PARAFAC)与多元线性回归(MLR)相结合, 对人 体三层皮肤模型的蒙特卡罗模拟实验和葡萄糖水溶液及其混合物的离体实验进行了验证. 实验结果表明，与传统的单 一母体背景所建立的偏最小二乘模型相比, 将母体背景作为建模元素采用 PARAFAC-MLR法所建立的校正模型具有更 好的预测能力和稳健性.
\end{abstract}

关键词 近红外；平行因子分析；多元线性回归；蒙特卡罗；葡萄糖；背景

\section{Enhanced Robustness of Calibration Models Using Parallel Factor (PARAFAC) Analysis with NIR Spectral Data for Non-invasive Blood Glucose Monitoring}

\author{
Zhang, Wanjie Liu, Rong* Xu, Kexin \\ (State Key Laboratory of Precision Measuring Technology and Instruments, Tianjin University, Tianjin 300072)
}

\begin{abstract}
The main cause of the low prediction accuracy in non-invasive blood glucose monitoring with near-infrared (NIR) spectroscopy is that the variations induced by the changes of the measuring system in the prediction data set are inconsistent with those in the calibration data set. In this paper, a method to improve the robustness of the calibration models is proposed, in which the information of the matrix background is introduced as a variable into the calibration data and the three-way tensor is used to build the regression model. The idea of constructing regression models based on the hybrid algorithms consists of two steps. The first is to build a parallel factor (PARAFAC) model with its second-order advantage and calculate the scores and loadings. Then a multivariate linear regression (MLR) calibration model is built from the PARAFAC sample scores combined with the reference concentration values for quantification purposes. For the validation and prediction, the PARAFAC loadings are used to calculate the predicted scores with the validation and prediction data sets, and then the predicted concentration values can be deduced from the MLR model. The proposed method has been successfully applied to two NIR spectroscopy experiments. One is a Monte-Carlo simulation experiment of skin. The changes of the absorption coefficients and scattering coefficients of dermis are considered as the variations of the matrix background. The other is an in vitro experiment including glucose, haemoglobin and albumin solutions and the mixed composition solutions. The determination coefficients and root mean square error of prediction (RMSEP) values obtained from the PARAFAC-MLR models are compared with those obtained from traditional chemometrics tools such as partial least squares (PLS). The results show that the PLS model cannot handle uncalibrated variations whereas the way of introducing the matrix background to generate tensor data and the regression method based on the combination of PARAFAC and MLR perform better in model robustness and prediction precision.
\end{abstract}

Keywords near-infrared spectroscopy; parallel factor (PARAFAC); multivariate linear regression (MLR); Monte-Carlo; glucose; background

\section{1 引言}

目前，糖尿病患者一般通过小型血糖仪实现血糖的 自我检测，但这种有创的测量方式会引起患者疼痛，并 伴有感染的危险，在很大程度上限制了患者监测血糖的 积极性. 因此, 开发一种无创伤的血糖检测方法对于糖 尿病患者群体具有十分重要的意义. 近红外光谱法以其
无痛无创伤、穿透力强、实现方便等优点被认为是最具 有应用前景的无创血糖测量方法之一 ${ }^{[1 \sim 6]}$, 其测量原理 是基于葡萄糖分子的 $\mathrm{C}-\mathrm{H}$ 和 $\mathrm{O}-\mathrm{H}$ 键在近红外区域的 倍频和合频吸收. 将一束近红外光入射到人体的特定部 位后接收其漫反射或漫透射光强，通过建立光强与葡萄 糖浓度之间的数学模型来实现人体血糖浓度的预 测 ${ }^{[7 \sim 10]}$.

* E-mail: rongliu@tju.edu.cn

Received April 12, 2013; published May 16, 2013.

Project supported by the National Natural Science Foundation of China (Nos. 60938002, 30900275) and the National High Technology Research and Development Program of China ('863' Program, No. 2012AA022602).

项目受国家自然科学基金(Nos. 60938002,30900275)、国家 863 计划(No. 2012AA022602)资助. 
光谱的采集过程对样本本身状态的稳定性要求非 常高, 通常需要严格控制实验条件保持样本及实验环境 的稳定, 以减少背景变化所引入的干扰. 但在实际测量 中, 经常会出现量测体系的变化, 即校正阶段和预测阶 段的量测体系不一致，比如不同个体之间、同一个体的 不同测量部位之间的差异，即使同一个体的同一测量部 位, 成分浓度也会随着时间或人体生理状态的变化而变 化. 特别是对于人体组织这种的复杂系统, 血糖在组织 内的含量非常小, 人体的正常生理活动, 甚至情绪波动, 都会直接导致光谱的变化, 这些影响通常在测量过程中 难以避免或消除. 而近红外光谱分析中常用的多元校正 方法是基于光谱矩阵中的所有变异, 对葡萄糖分子并不 具有特异性. 因此, 测量过程中量测体系的背景差别会 直接影响到光谱模型的精度和稳健性. 当这种背景变化 的幅度超出校正模型所能够覆盖的程度时, 模型就会失 效, 这也是近红外光谱无创血糖检测技术还没有真正实 现临床应用的原因之一。

由于不同的个体或者同一个体的不同部位包含着 特定的母体背景信息, 因此可以采用高阶数据分析方 法, 如平行因子法(parallel factor, PARAFAC) ${ }^{[11]}$, 将不 同的母体信息作为一个建模元素来参与回归计算. 这 样, 模型中除了包含待测成分浓度、光谱变量(波长或波 数)外, 还包含了母体的变异信息, 有助于提高分析模 型的性能. PARAFAC 是一种广义的高维主成分分析的 分辨方法, 其分解具有唯一性, 只要数据信噪比和选择 的因子数合适, 分解的结果就能反映出真实的数据变 异. 它具有预测精度高、稳健性强、光谱解释力优等特 点, 其最大的优点是具有显著的二阶校正优势(secondorder advantage), 即使预测集中存在校正集不存在的干 扰物质, PARAFAC 分析结果也不会明显恶化, 并且不 受光谱的非线性变化的影响 ${ }^{[12,13]}$. 该方法已被广泛应用 于生物或化学计量学领域, 如 Peinado 等 ${ }^{[14]}$ 采用 PARAFAC 对受温度影响的近红外光谱回归分析, 可有 效解决由温度变化带来的非线性影响; Alm 等 ${ }^{[15]}$ 将其用 于 IR 和 NIR 的光谱信息融合; Cordella 等 ${ }^{[16]}$ 采用 PARAFAC 对小麦面条的化学成分, 感官属性和评估标 准(assessor)等三个维度进行建模, 实现了对不同类型面 条的有效区分, 等等.

本文主要采用PAFAFAC 分析方法结合多元线性回 归(multivariate linear regression, MLR)分别对两组近红 外光谱数据进行了分析, 一组为人体三层皮肤模型的蒙 特卡罗模拟实验, 以真皮层的吸收系数和散射系数的变 动来模拟母体背景的变动; 另一组为葡萄糖水溶液的离 体实验, 引入血红蛋白和白蛋白来模拟母体背景的变 动. 将 PARAFAC-MLR 模型预测结果的预测均方根误 差(root mean standard error of prediction, RMSEP)与单一 背景所建立的 PLS 模型结果比较, 验证了该方法在提高 校正模型稳健性及抗干扰能力方面的有效性.

\section{2 方法原理}

通常, 在近红外光谱分析中, 每个样本在 $J$ 个变量 (波长或波数)下形成一个光谱向量, $I$ 个样本则形成一个 光谱矩阵 $\boldsymbol{X}_{(I \times J)}$. 在无创血糖检测中, 不同的个体之间或 同一个体的不同测量部位之间具有不同的光学性质，即 使相同的葡萄糖浓度，在不同的背景下所得到的光谱也 不尽相同. 因此, 将 $K$ 个不同背景下测得的光谱矩阵汇 在一起，即可构成一个三阶张量(tensor) $\underline{X}_{(I \times J \times K)}$.

PARAFAC-MLR 方法的分析流程如图 1 所示. 首先 对校正集光谱立体阵进行 PARAFAC 分析, 将其分解为 $N$ 组三变量组, 表示成统计学形式为:

$$
\begin{aligned}
& x_{i j k}=\sum_{n=1}^{N} a_{i n} b_{j n} c_{k n}+e_{i j k} \\
& i=1,2, \ldots, I ; j=1,2, \ldots, J ; k=1,2, \ldots, K
\end{aligned}
$$

其中, $x_{i j k}$ 表示 $i$ 个样本在变量 $j$ 和 $k$ 下的值, 是主成分 数为 $N$ 的三线性数据阵, 即立体阵 $\underline{X}_{(I \times J \times K)}$ 的元素, $a_{i n}, b_{j n}, c_{k n}$ 是分解所得到的成分矩阵的元素, 分别对 应一个得分矩阵和两个载荷矩阵. $e_{i j k}$ 是残差立体阵 $\underline{E}_{(I \times J \times K)}$ 的元素. PARAFAC 分解的主要目的是使得残差 $e_{i j k}$ 的平方和最小 ${ }^{[17]}$. 进一步, 建立得分矩阵(即第一维) 与已知样品的参考浓度之间的 MLR 校正模型. 对于预 测集光谱矩阵，利用校正集 PARAFAC 分解得到的两个 载荷矩阵(即第二维和第三维)进行 PARAFAC 分析, 得

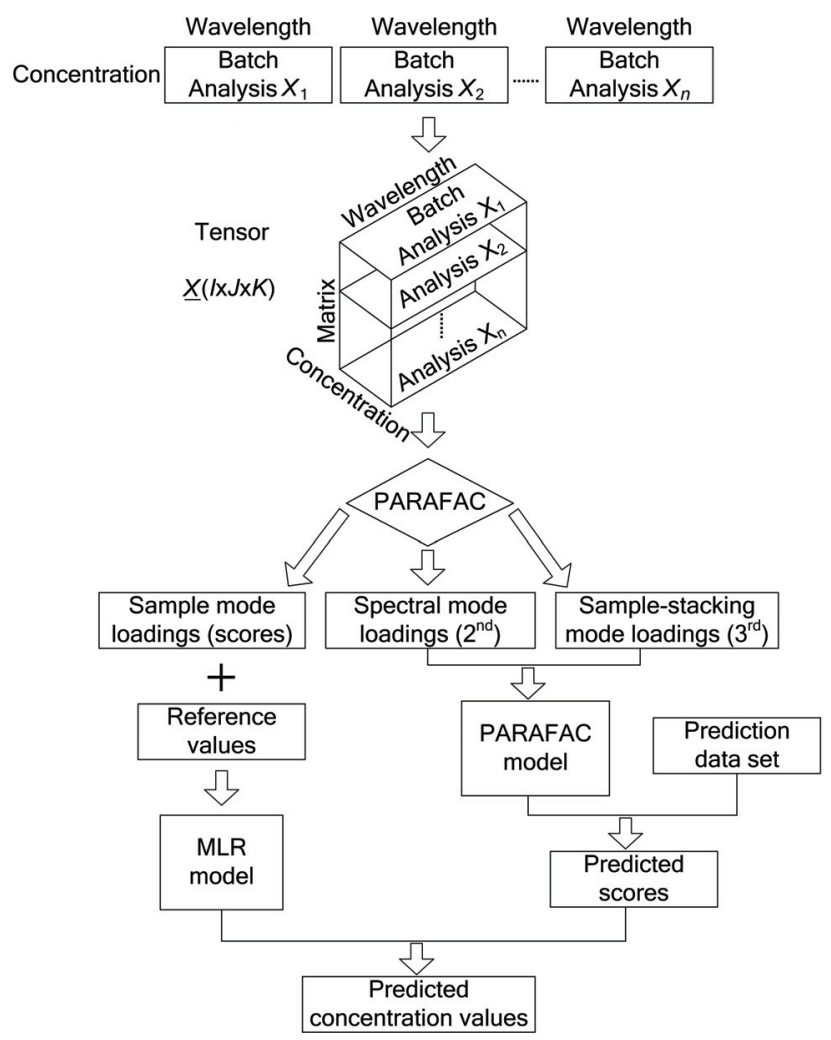

图 1 光谱矩阵的 PARAFAC-MLR 分析流程图

Figure 1 Diagram of the theory of PARAFAC-MLR 
到估计的得分矩阵, 进而利用所建立的 MLR 回归模型 对估计的得分矩阵进行预测, 即可得到待分析成分的浓 度.

\section{3 结果与讨论}

\section{1 蒙特卡罗模拟实验}

经过对人体三层皮肤模型的蒙特卡罗模拟，从得到 的 25 组光谱数据中选取三组典型的光谱数据, 吸收系 数 $\mu_{a}$ 和约化散射系数 $\mu_{s}{ }^{\prime}$ 组合分别为 $\mu_{a 0}$ 和 $\mu_{s 0}{ }^{\prime}$, $-20 \% \mu_{a 0}$ 和 $-20 \% \mu_{s 0}{ }^{\prime} ， 20 \% \mu_{a 0}$ 和 $20 \% \mu_{s 0}{ }^{\prime}$, 分别记 作数据集 1,2 和 3 , 每组数据集均为 10 (样本) $\times 26$ (波 长)的光谱矩阵. 以这三组光谱矩阵作为校正集建立三 个 PLS 模型, 为 PLS-1, PLS-2 和 PLS-3, 分别采用 “留 一法” 的完全交互验证(leave-one-out cross validation)对 这三个校正模型的性能进行评价, 再分别以其余两组光 谱矩阵作为预测集，对其中的某一个校正模型的预测性 能进行评价. 模型的预测性能以预测值与真实值之间的 RMSEP 值和决定系数 $R^{2}$ (determination coefficient) 作为 评价参数:

$$
\begin{aligned}
& R M S E P=\sqrt{\frac{\sum_{i=1}^{n}\left(\hat{y}_{i}-y_{i}\right)^{2}}{n-1}} \\
& R^{2}=\frac{\sum_{i=1}^{n}\left(\hat{y}_{i}-y_{i}\right)^{2}}{\sum_{i=1}^{n}\left(\overline{\hat{y}}_{i}-y_{i}\right)^{2}}
\end{aligned}
$$

式中, $y_{i}$ 为待测成分浓度的真实值, $\hat{y}_{i}$ 为模型的预测结 果, $\overline{\hat{y}}_{i}$ 为 $\hat{y}_{i}$ 的均值, $n$ 为待测样本数. 采用 PLS 建立的 校正模型的预测结果如表 1 所示.

表 1 三个 PLS 模型校正及预测分析结果

Table 1 Regression and prediction results of the three PLS models

\begin{tabular}{ccccccc}
\hline \multirow{2}{*}{ Models } & \multicolumn{2}{c}{$\begin{array}{c}\text { Leave-one-out } \\
\text { cross validation }\end{array}$} & & \multicolumn{2}{c}{$\begin{array}{c}\text { Prediction sets validation } \\
{\left[\mathrm{RMSEP} /\left(\mathrm{mmol} \cdot \mathrm{L}^{-1}\right)\right]}\end{array}$} \\
\cline { 2 - 3 } \cline { 5 - 7 } & $R^{2}$ & $\begin{array}{c}\mathrm{RMSEP} / \\
\left(\mathrm{mmol} \bullet \mathrm{L}^{-1}\right)\end{array}$ & & 1 & 2 & 3 \\
\hline PLS-1 & 0.99972 & 1.12 & & - & 6.70 & 9.28 \\
$P L S-2$ & 0.99959 & 1.05 & & 6.33 & - & 13.84 \\
$P L S-3$ & 0.99979 & 0.86 & & 9.40 & 15.64 & - \\
\hline
\end{tabular}

从表 1 中可以看出, 采用完全交互验证时, 三个校 正模型的预测精度都较高，决定系数均达到 $99.9 \%$ 以上; 但当采用独立预测集时, 各个模型的预测精度都明显降 低. 原因可以解释为预测集与校正集的光谱矩阵中的光 学参数存在差异(可以认为是不同生理时刻或者不同个 体背景存在差异), 而这些差异导致了校正模型无法涵 盖预测集样本的变化特征，从而不能有效提取预测集中 葡萄糖变化信息，造成预测精度较低.

将模拟得到的全部 25 组不同情况的 $\mu_{a}$ 和 $\mu_{s}^{\prime}$ 组合
作为 25 个切片 (slab), 结合各个切片下的 10 (样本) $\times$ 26(波长)的光谱矩阵, 构建一个浓度-波长一背景的三维 张量, 采用图 1 所示的流程对得到的张量进行 PARAFAC 分解得到第一载荷(得分)矩阵, 并结合葡萄 糖浓度建立 MLR 模型, 记作 PARAFAC-MLR-all. 进一 步, 将这 25 组不同的 $\mu_{a}$ 和 $\mu_{s}{ }^{\prime}$ 组合的光谱矩阵随机分 成两部分, 一部分包含 13 组组合(即不同情况), 作为 13 个切片, 结合各个切片下的光谱矩阵构成一个张量(记 作数据集 $Y$ ), 首先以该张量作为校正集进行 PARAFAC 分解得到第一载荷(得分)矩阵, 结合葡萄糖 浓度构建一个 MLR 模型，记作 PARAFAC-MLR- $\underline{Y}$ 。剩 余的 12 组组合构成 12 个切片，结合各个切片下的光谱 矩阵同样构建一个张量(记作数据集 $\underline{Z}$ ). 下面以数据集 $\underline{Y}$ 和 $\underline{Z}$ 分别作为预测集，代入两个 PARAFAC 模型中, 得到预测得分，结合已建立的 MLR 模型，得到预测浓 度值, 结果如表 2 所示. 采用全部光谱数据所建立的校 正模型，已经涵盖了预测集中的光谱变动特征，因此可 以获得理想的预测精度，预测值与真值之间具有良好的 线性关系, RMSEP 值明显优于 PLS 模型对预测集的预 测结果; 而光谱数据集 $\underline{Y}$ 与 $\underline{Z}$ 之间所包含的母体背景 并不相同, 采用数据集 $\underline{Y}$ 对含有未知干扰的预测集 $\underline{Z}$ 进行预测时，由于 PARAFAC 具有二阶特性，抗干扰能 力强, 同样能够得到较好的预测结果. 另外, 从表 2 中 也可以看到，当采用同一模型对不同的数据集进行预测 时, 得到的预测精度略有差异, 这是因为 $\underline{Y}$ 和 $\underline{Z}$ 是由全 部数据随机分配的两组数据集，它们的数据量大小不同 而且数据本身存在差异. 当采用 $P A R A F A C-M L R$-all 模 型对数据集 $\underline{Y}$ 和 $\underline{Z}$ 进行预测时，尽管模型覆盖了预测 集光谱中的变化特征，但由于两预测集本身数据不同从 而造成得到的 RMSEP 值略有不同. 而采用 PARAFAC-MLR- $\underline{Y}$ 模型对两数据集进行预测时, 由于模 型覆盖了预测集 $\underline{Y}$ 中的光谱变化特征，而预测集 $\underline{Z}$ 中 含有模型中不包含的未知干扰，因此对数据集 $Y$ 的预测 精度要优于对数据集 $\underline{Z}$ 的预测精度. 同样地，采用两个 不同的模型对同一数据集进行预测时, 得到的预测精度 也略有差异，这是因为所建立的模型对预测集的光谱变 化的覆盖程度不同造成的. 采用 PARAFAC-MLR-all 模 型和 $P A R A F A C-M L R-\underline{Y}$ 模型分别对预测集 $\underline{Y}$ 进行预测, 虽然均可以获得较为理想的预测精度, 但由于 PARAFAC-MLR-all 模型相对于 PARAFAC-MLR- $\underline{Y}$ 模型 中包含有更多的除数据集 $\underline{Y}$ 外的光谱变化信息, 在模型 稳健性提高的同时，以预测精度的降低为代价. 采用两 个模型对预测集 $\underline{Z}$ 进行预测的结果类似.

\section{2 离体实验}

测量葡萄糖、葡萄糖和白蛋白的混合物、葡萄糖和 血红蛋白的混合物三组水溶液的透射光谱, 得到三组吸 光度光谱矩阵, 将三组矩阵结合构成葡萄糖浓度-波长一 混合物的三维张量, 采用核一致诊断法(core consistency 
表 2 PARAFAC-MLR 模型预测分析结果

Table 2 Prediction results of the PARAFAC-MLR models

\begin{tabular}{|c|c|c|c|c|}
\hline \multirow{3}{*}{ Models } & \multicolumn{4}{|c|}{ Prediction results } \\
\hline & \multicolumn{2}{|r|}{$\underline{Y}$} & \multicolumn{2}{|r|}{$\underline{Z}$} \\
\hline & $R^{2}$ & $\begin{array}{c}\text { RMSEP/ } \\
\left(\mathrm{mmol} \cdot \mathrm{L}^{-1}\right)\end{array}$ & $R^{2}$ & $\begin{array}{c}\text { RMSEP/ } \\
\left(\mathrm{mmol} \cdot \mathrm{L}^{-1}\right)\end{array}$ \\
\hline PARAFAC-MLR-all & 0.99969 & 2.21 & 0.99988 & 1.55 \\
\hline PARAFAC-MLR- $\underline{Y}$ & 0.99994 & 1.04 & 0.99907 & 3.09 \\
\hline
\end{tabular}

diagnostic, CORCONDIA) ${ }^{[12,13]}$ 对该三维数据进行分析, 当预估计因子数为 4 时, 函数值接近于 $100 \%$, 因此取主 因子数为 4 进行 PARAFAC 分析, 得到三个维度的载荷 分别如图 2(A C) 所示, 分别对应样本维 $(A)$ (sample mode)、光谱维(B) (spectral mode)和样本堆维(C) (sample-stacking mode). 对比图 2(A)和图 3 中的样本中的浓 度图可以发现, 4 个因子的曲线分别与血红蛋白、白蛋 白、水和葡萄糖浓度曲线相似. 对比图 2(B)与图 2(D)的 4 种成分的摩尔吸光系数曲线可以看出, 4 个因子的曲线 分别包含 4 种成分各自的特征吸收峰, 如第一因子在波 长低于 $1200 \mathrm{~nm}$ 附近存在血红蛋白的吸收峰, 第二因子 在波长 $1430 \mathrm{~nm}$ 附近存在白蛋白的吸收峰等. 图 2(C)则
对应样本堆维，以第一因子为例，其在葡萄糖、葡萄糖 和白蛋白处值约为 0 , 而在葡萄糖和血红蛋白处为最大 值，其余三个因子具有类似的关系.

将测量得到的葡萄糖、葡萄糖和白蛋白的混合溶 液、葡萄糖和血红蛋白的混合溶液三组吸光度光谱矩阵 分别作为校正集建立 PLS 模型, 并以其他两组光谱矩阵 作为预测集对其进行预测, 结果如表 3 所示. 当预测集 样本与校正集样本不再保持一致或存在校正集中不存 在的干扰成分时, 此时所建立的校正模型则完全失去了 预测能力. 即使采用葡萄糖、血红蛋白和白蛋白的混合 溶液的吸光度光谱数据建立 PLS 模型时, 由于干扰成分 浓度高、吸收强，部分掩盖了葡萄糖浓度的有效信息， 因此采用该模型对另外三种吸光度光谱数据进行预测 时, 得到的预测结果也不甚理想. 采用上述由葡萄糖、 葡萄糖和白蛋白的混合物、葡萄糖和血红蛋白的混合物 的吸光度光谱矩阵所构成的葡萄糖浓度-波长一混合物 的三维张量作为校正集，根据图 1 所示的方法建立 PARAFAC-MLR 校正模型, 以三组吸光度光谱矩阵分 别作为预测集进行预测, 得到的 RMSEP 值分别为 $37.46,62.52$ 和 $48.39 \mathrm{mg} / \mathrm{dL}$, 与 PLS 模型预测得到的
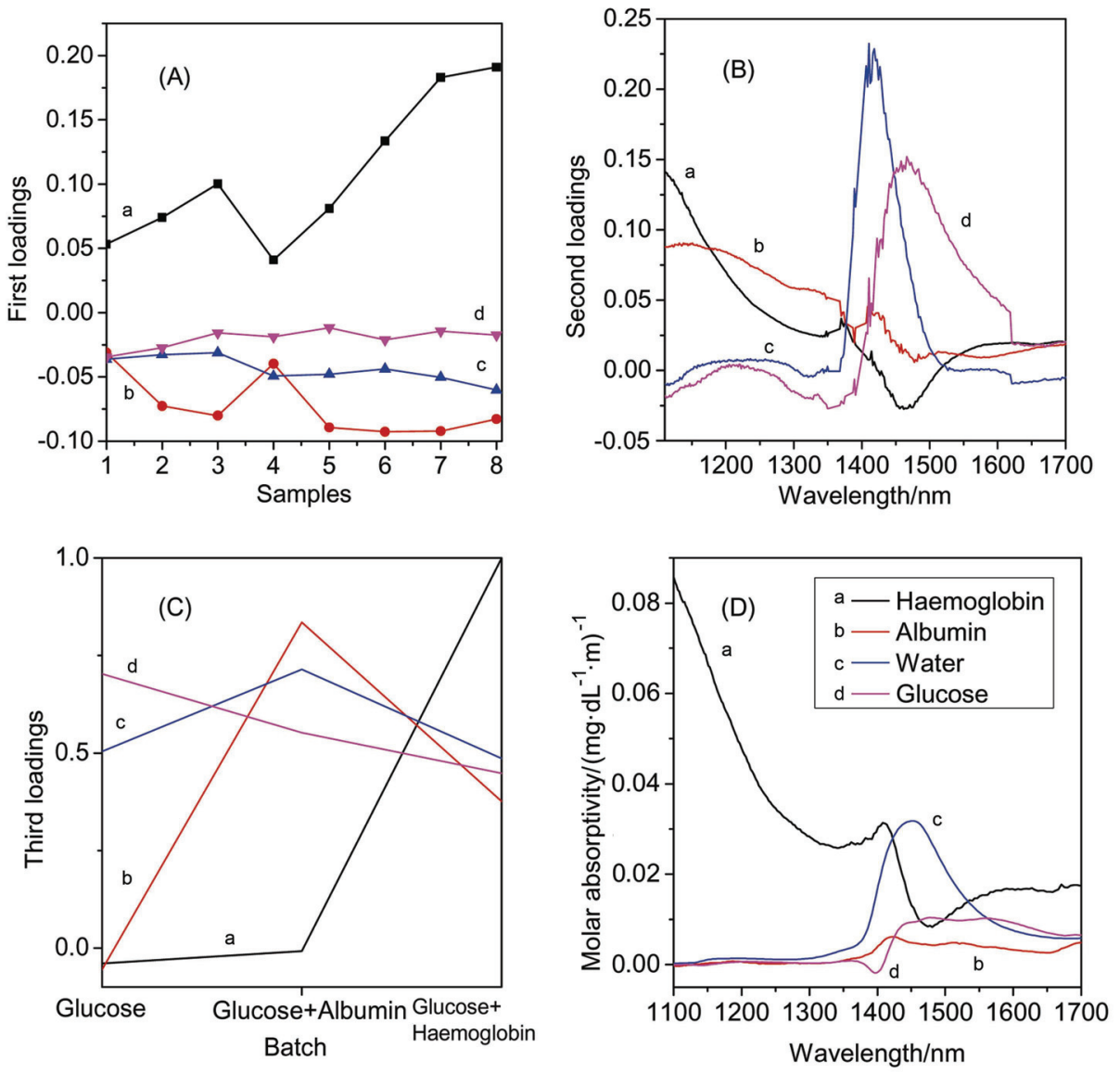

图 2 三组混合物 PARAFAC 分析结果

Figure 2 Results of PARAFAC analysis of the mixed solutions

(a) = factor $1,(\mathrm{~b})=$ factor $2,(\mathrm{c})=$ factor 3 , (d) = factor 4 : (A) sample loadings $\left(1^{\text {st }}\right.$ mode $)$; (B) spectral loadings $\left(2^{\text {nd }}\right.$ mode $)$; (C) sample-stacking loadings $\left(3^{\text {rd }}\right.$ mode); (D) molar absorptitvities of haemoglobin (a), albumin (b), water (c), and glucose (d) 
表 3 PLS 和 PARAFAC 模型预测结果对比

Table 3 Prediction results of the PLS and PARAFAC-MLR models

\begin{tabular}{cccc}
\hline \multirow{2}{*}{ Model } & \multicolumn{3}{c}{$\begin{array}{c}\text { Glucose prediction results [RMSEP/ } \\
\left.\left(\mathrm{mg} \bullet \mathrm{dL}^{-1}\right)\right]\end{array}$} \\
\cline { 2 - 4 } & $\mathrm{G}$ & $\mathrm{G} \& \mathrm{~A}$ & $\mathrm{G} \& \mathrm{H}$ \\
\hline PLS-G $^{a}$ & 43.03 & 478.18 & 545.88 \\
PLS-G\&A $^{b}$ & 355.81 & 39.64 & 682.59 \\
PLS-G\&H $^{c}$ & 276.86 & 199.92 & 15.54 \\
PLS-G\&H\&A & 382.53 & 161.27 & 240.19 \\
PARAFAC & 37.46 & 62.52 & 48.39 \\
\hline
\end{tabular}

${ }^{a} \mathrm{G}$ refers glucose; ${ }^{b} \mathrm{G} \& \mathrm{~A}$ refers the mixture of glucose and albumin; ${ }^{c} \mathrm{G} \& \mathrm{H}$ refers the mixture of glucose and haemoglobin; ${ }^{d} \mathrm{G} \& H \& A$ refers the mixture of glucose, haemglobin, and albumin.

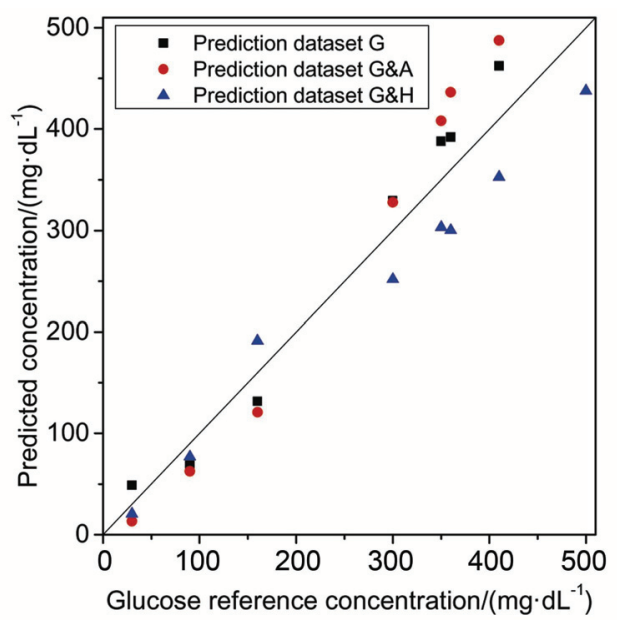

图 3 PARAFAC-MLR 模型对三组预测集数据的葡萄糖浓度预测值 与参考值的相关曲线

Figure 3 Concentration correlation plots for PARAFAC-MLR model for glucose

solid line corresponds to the ideal, unity correlation between prediction and reference concentrations

RMSEP 相比降低一个量级, 预测精度得到了明显的提 高，预测结果与参考真实浓度值之间的关系曲线如图 3 所示, 三组预测集所得到的决定系数分别为 0.9946 , 0.9961 和 0.9897. 另外, 从表 3 中看出, 与预测集和样本 集吸光度光谱为同一数据集时所建立的 PLS 模型的预 测结果相比, PARAFAC-MLR 校正模型的 RMSEP 值略 高, 模型的稳健性提高, 但是预测精度降低. 这是因为 建模时所采用的数据信息增大，提高了模型的稳健性， 但与此同时, 干扰信息也增加了, 降低了模型的分析精 度. 但是由于 PARAFAC 分析方法的二阶优势, 使得模 型仍能获得较为理想的预测结果.

\section{4 结论}

本文主要采用 PARAFAC 建模方法对提高近红外 光谱校正模型的稳健性和预测性能进行了研究和探讨. 在近红外光谱无创血糖检测过程中, 母体或组织的变动 会对预测结果产生显著影响, 因此将母体或组织的变动
特性作为变量引入校正模型中参与回归计算, 构建待测 成分浓度-光谱变量-母体变异信息的多维数据, 采用 PARAFAC 与 MLR 相结合的化学计量学方法, 提高分 析模型的稳健性和预测能力. 本文分别进行了人体三层 皮肤模型的蒙特卡罗模拟实验和葡萄糖水溶液及其混 合物的离体实验, 实验结果表明, 采用 PARAFAC-MLR 方法所建立的校正模型的预测能力明显优于采用单一 母体背景所建立的 PLS 模型, 提高了模型的稳定性. 在 下一步的工作中，可以将该校正模型用于对真实血浆或 全血样品数据的预测, 以考察 PARAFAC-MLR 模型的 预测结果与实际分析结果的偏离程度. 另外, 可以将其 他的变异因素(如测量条件的变化等)引入模型中, 建立 更高维的数据模型.

\section{5 实验部分}

\section{1 蒙特卡罗模拟实验}

当光入射到组织表面时, 在组织内主要发生吸收效 应和散射效应，不同母体或组织的差别和变化最终都体 现在组织光学特性的差异之中. 如血红蛋白等成分浓度 增加, 主要影响的是吸收系数 $\mu_{a}$; 脂肪等浓度变化则 主要影响组织的散射系数 $\mu_{s}$. 由于散射效应在组织体 内葡萄糖浓度检测中占主要地位，常采用漫射近似，此 时采用约化散射系数 $\mu_{s}^{\prime}$ 来描述组织的散射特性 $\left(\mu_{s}^{\prime}=\mu_{s} \times(1-g)\right.$, 其中 $g$ 表示各向异性因子).

采用 Wang 等 ${ }^{[18]}$ 发展的用于多层组织内稳态光的传 播过程模拟和特性参数计算的 Monte Carlo 程序, 模拟 人体三层皮肤的光传输过程, 获取漫反射光的分布特 性, 其中各波长下的各层皮肤组织的光学参数来自 Maruo ${ }^{[5,19]}$. 通过改变真皮层的 $\mu_{a}$ 和 $\mu_{s}{ }^{\prime}$ ，来模拟不同母 液或组织，或者是同一母液或组织在不同时刻下的状 态，变化范围分别为 $-20 \% \sim 20 \%$ (梯度为 $10 \%$ ), 得到共 25 组不同的 $\mu_{a}$ 和 $\mu_{s}{ }^{\prime}$ 的组合. 模拟以无限细点光源入 射, 光源波长变化范围为 $1200 \sim 1700 \mathrm{~nm}$, 变化梯度为 $20 \mathrm{~nm}$, 采用入射光子总数为 $10^{8}$ 个. 在每组 $\mu_{a}$ 和 $\mu_{s}{ }^{\prime}$ 的 组合下, 改变葡萄糖浓度, 变化范围为 $0 \sim 200 \mathrm{mmol} / \mathrm{L}$, 变化梯度为 $20 \mathrm{mmol} / \mathrm{L}$. 将各波长下距离光源径向距离 为 $0.5 \mathrm{~mm}$ 位置的漫反射光扣除不含葡萄糖的漫反射光, 并取负对数，得到每组 $\mu_{a}$ 和 $\mu_{s}{ }^{\prime}$ 的组合下各浓度的吸光 度光谱各 10 条.

\section{2 离体实验}

采用美国 Perkin-Elmer 公司的 Spectrum GX 傅里叶 变换红外光谱仪, 分别测量四组含有葡萄糖的水溶液样 本的近红外光谱, 每组包含 8 个样本, 葡萄糖的浓度在 10 500 mg/dL 范围内随机分布. 四组样本分别为: (1) 葡萄糖的水溶液; (2) 葡萄糖和白蛋白混合物的水溶液, 其中白蛋白浓度范围为 $0 \sim 200 \mathrm{mg} / \mathrm{dL}$; (3) 葡萄糖和血红 蛋白混合物的水溶液, 其中血红蛋白浓度范围为 200 $1000 \mathrm{mg} / \mathrm{dL}$; (4)葡萄糖、血红蛋白和白蛋白混合物的水 
溶液. 4 组样品中的葡萄糖浓度相同, 其中各组样品中 葡萄糖、白蛋白和血红蛋白的浓度如图 4 所示. 实验中 对每组的 8 个样本采取随机抽取顺序进行光谱数据采 集, 采集每个样本的光谱后立即测量纯水的透射光谱作 为背景光谱, 共测量 32 个 $(8 \times 4)$ 样本的透射光谱和 32 个纯水背景光谱. 将样本光谱除以背景光谱并取负对数 运算, 得到的吸光度值进行相应的数据预处理. 为去除 不同组样本光谱矩阵自身信号强度量级的影响, 对得到 的吸光度光谱矩阵分别进行归一化处理, 将不同样本的 归一化后的矩阵用于建模和预测分析.

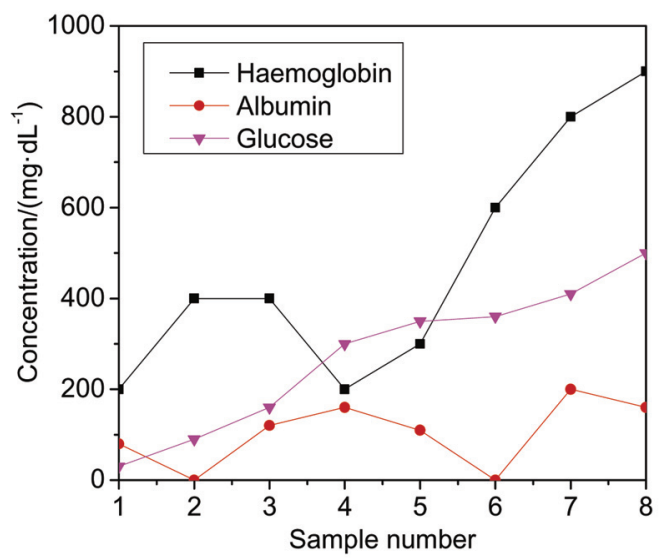

图 4 实验样本中成分浓度

Figure 4 Concentrations of the components

\section{3 数据分析与软件}

MLR 校正模型利用 CAMO 公司的 Unscrambler 9.7 软件建立, PARAFAC 程序来自: http://www.models.life.ku.dk/source/nwaytoolbox $/ /^{[20]}$.

\section{References}

[1] Malin, S. F.; Ruchti, T. L.; Blank, T. B.; Thennadil, S. N.; Monfre, S. L. Clin. Chem. 1999, 45, 1651.

[2] Klonoff, D. C. Diabetes Care 1997, 20, 433.

[3] Arnold, M. A.; Small, G. W. Anal. Chem. 2005, 77, 5429.

[4] Kondepati, V. R.; Heise, H. M. Anal. Bioanal. Chem. 2007, 388, 545.

[5] Maruo, K.; Tsurugi, M.; Chin, J.; Ota, T.; Arimoto, H.; Yamada, Y.; Tamura, M.; Ishii, M.; Ozaki, Y. IEEE J. Sel. Top. Quantum Electron. 2003, 9, 322.

[6] Arnold, M. A.; Liu, L.-Z.; Olesberg, J. T. J. Diabetes Sci. Technol. $2007,1,454$.

[7] Olesberg, J. T.; Liu, L.-Z. Anal. Chem. 2006, 78, 215.

[8] Ozaki, Y. Anal. Sci. 2012, 28, 545.

[9] Shao, X.-G.; Ning, Y.; Liu, F.-X.; Li, J.-H.; Cai, W.-S. Acta Chim. Sinica 2012, 70, 2109. (邵学广, 宁宇, 刘风霞, 李积慧, 蔡文生, 化学学报, 2012, 70, 2109.)

[10] Chu, X.-L. Molecular Spectroscopy Analytical Technology Combined with Chemometrics and Its Application, Chemical Industry Press, Beijing, 2011. (禇小立, 化学计量学方法与分子光谱分析 技术, 化学工业出版社, 北京, 2011.)

[11] Shinzawa, H.; Nishida, M.; Kanematsu, W.; Tanaka, T.; Suzuki, K.; Noda, I. Analyst 2012, 137, 1913.

[12] Andersson, C. A.; Bro, R. Chemom. Intell. Lab. Syst. 2000, 52, 1.

[13] Bro, R. Chemom. Intell. Lab. Syst. 1997, 38, 149.

[14] Peinado, A. C.; van den Berg, F.; Blanco, M.; Bro, R. Chemom. Intell. Lab. Syst. 2006, 83, 75 .

[15] Alm, E.; Bro, R.; Engelsen, S. B.; Karlberg, B.; Torgrip, R. J. O Anal. Bioanal. Chem. 2007, 388, 179.

[16] Cordella, C. B. Y.; Leardi, R.; Rutledge, D. N. Chemom. Intell. Lab. Syst. 2011, 106, 125.

[17] Wang, K.; Jia, Z.-H.; Zhang, Z.-Q.; Li, H. Chem. J. Chin. Univ. 2009, 30, 268. (王康, 贾泽慧, 张志琪, 李华, 高等学校化学学报, 2009, 30, 268.)

[18] Wang, L.-H.; Jacques, S. L.; Zheng, L. Q. Comput. Meth. Prog. Bio. $1995,47,131$.

[19] Maruo, K.; Oota, T.; Tsurugi, M.; Nakagawa, T.; Arimoto, H.; Tamura, M.; Ozaki, Y.; Yamada, Y. Appl. Spectrosc. 2006, 60, 441.

[20] http://www.models.life.ku.dk/source/nwaytoolbox/ 